\title{
Monitoring ADHD in treatment with a new biological marker of ADHD
}

\section{Paloma Varela1, Josep Antoni Ramos-Quiroga², Hans Supèr ${ }^{3-7}$ Jose Cañete $^{1}$}

1) Mental Health Dept, Consorci Sanitari del Maresme, Mataró, Spain; 2) Department of Psychiatry, CIBERSAM, Hospital Universitari Vall d'Hebron, Barcelona, Spain; 3) Braingaze SL, Mataró, Spain; 4) Departament de Cognició, Desenvolupament i Psicologia de l'Educació, Faculty of Psychology, University of Barcelona, Barcelona, Spain; 5) Institut de Neurociències, Universitat de Barcelona, Barcelona, Spain 6) Institut de Recerca Pediàtrica Hospital Sant Joan de Déu, Barcelona, Spain; 7) ICREA , Barcelona, Spain

Contact hans.super@icrea.cat

Objectives: Recently we described a new method for diagnosing ADHD in children. The method consist of recording miniature eye movements (1), which are implicated in attentional processing. We showed that these eye movement are absent or weak in ADHD children (2) and can be used as an objective biological marker for ADHD (called Cognitive Vergence). In order to know whether the biological maker can be useful for monitoring treatment, we investigated the possible effect of typical pharmaceutical intervention to reduce ADHD symptoms on the level of the biological marker.

Methods: After extensive clinical diagnosis some children $(\mathrm{N}=14 ; 7-14$ years of age) with ADHD received pharmaceutical treatment (Methylphenidate or Lisdexamfetamine Dimesylate) while others $(\mathrm{N}=7)$ did not receive pharmaceutical medication. Before treatment all ADHD children performed the BGaze test (Braingaze, Spain) to assess the probability and severity of ADHD based on the level of biological marker (Pre). Six to eight months after treatment or the initial diagnosis these children were re-tested with the BGaze test (Post). For statistics we applied Student t-tests.

Results: Preliminary results (mean \pm std) of this pilot suggests a lowering of the severity score measured by the biological marker in the medicated group (Pre: $0.46 \pm 0.24$; Post: $0.38 \pm 0.20$; $\mathrm{p}=0.08$ ) and not in the non-medicated group (Pre: $0.36 \pm 0.34$; Post: $0.34 \pm 0.14 ; p=0.46)$. The reduction in severity score was significantly stronger $(P<0.001)$ in the medicated group than in the non-medicated group. The probability scores the remained similar (Medicated group: Pre: $0.55 \pm 0.08$; Post: $0.56 \pm 0.08 ; \mathrm{p}=0$ 38; Non-medicated group: Pre: $0.53 \pm 0.20$; Post: $0.54 \pm 0.15$; $\mathrm{p}=0.39$ ).

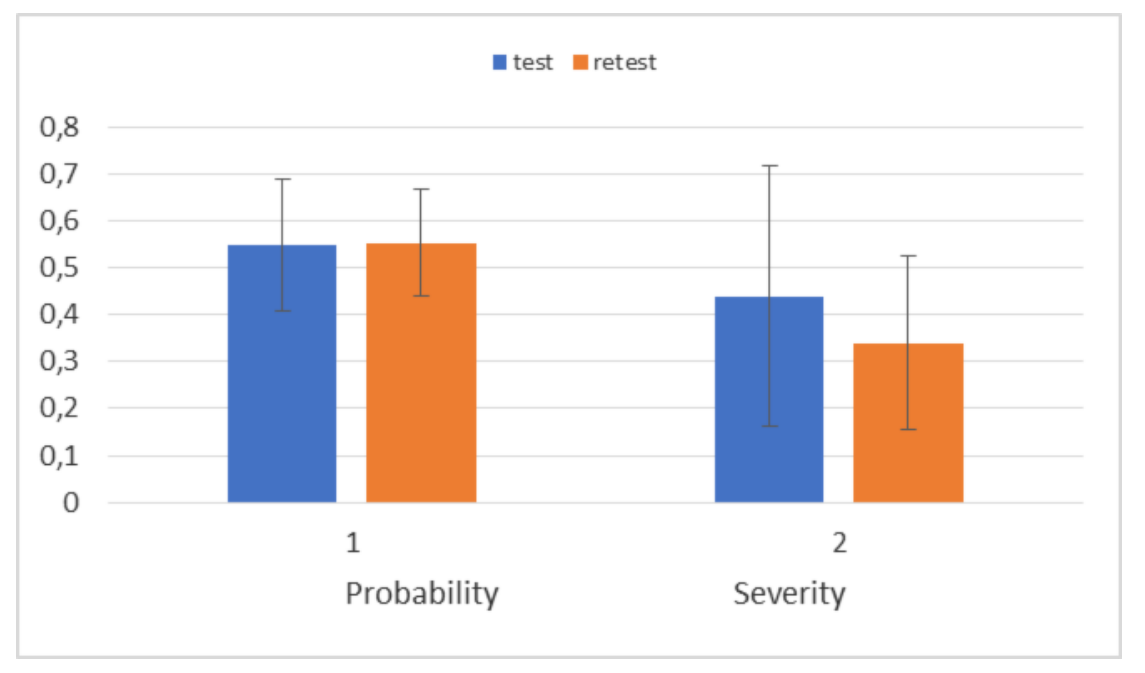

Average probability and severity scores obtained from the neural marker test from BGaze at pre test and post test times for children with and without medication

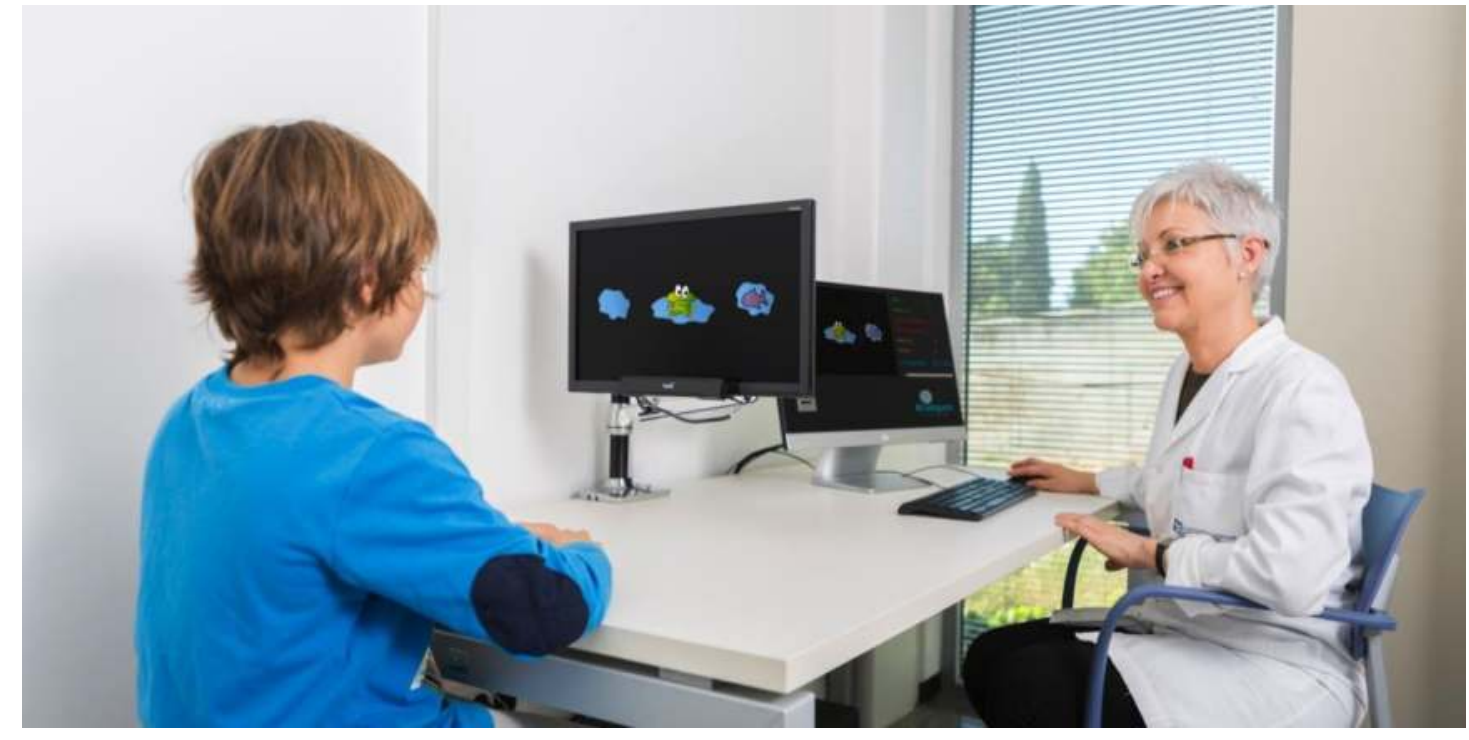

BGaze system with neural marker test for diagnosis of ADHD

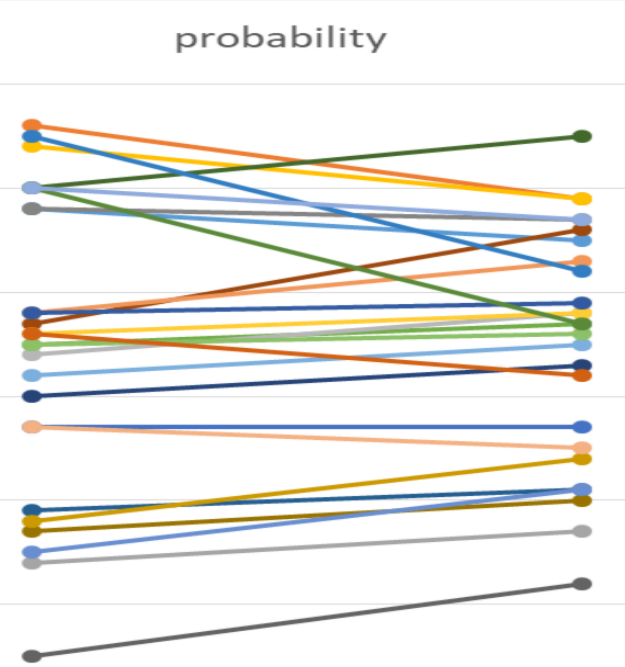

Probability of having ADHD at pre test and post test times for children with and without medication

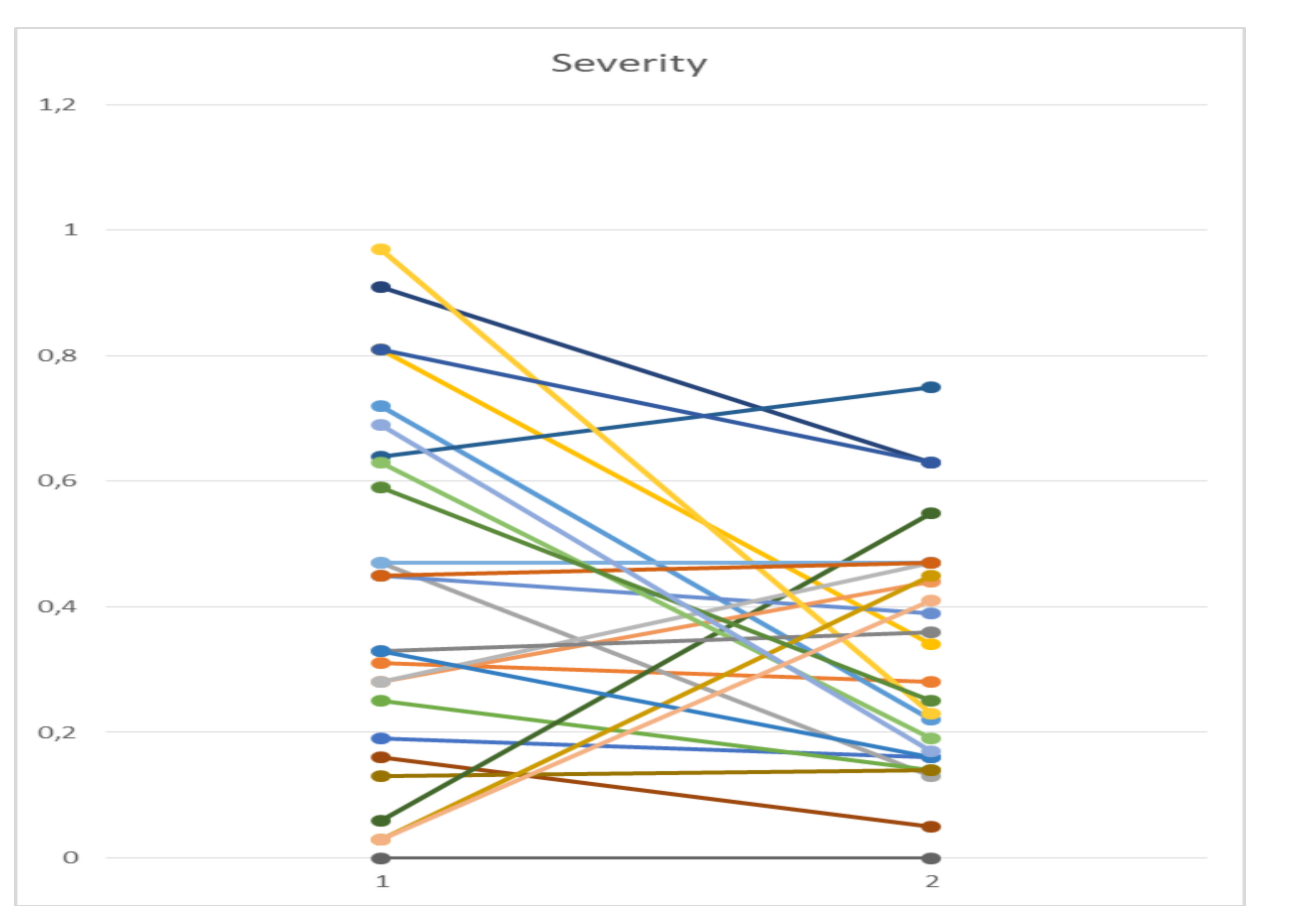

Severity scores obtained from the neural marker test from BGaze at pre test and post test times for children with and without medication

Conclusions. These findings suggest that pharmaceutical intervention lowers the severity of ADHD measured by the biological marker (Cognitive Vergence) of the BGaze test. A study (currently undertaken) with larger patient sample is needed to confirm whether Cognitive Vergence assessmentcan be used as a tool to monitor pharmaceutical treatment. 